\title{
ON SEMICONTINUOUS LINEAR LATTICES
}

\author{
HIDEGORO NAKANO
}

Abstract. Applying spectral theory, we proved that a linear lattice is continuous if and only if it is semicontinuous and uniformly complete. In this paper we give another proof without use of spectral theory.

Let $L$ be a linear lattice. A sequence of elements $x_{v} \in L(\nu=1,2, \cdots)$ is called a uniform Cauchy sequence if there is $a \in L$ such that for any $\varepsilon>0$ we can find $v_{0}$ for which $\left|x_{\mu}-x_{v}\right| \leqq \varepsilon|a|$ for $\mu, v \geqq v_{0}$. L is said to be uniformly complete if every uniform Cauchy sequence is convergent. We can easily prove that every uniformly complete linear lattice is Archimedean, as done in [1].

A linear lattice $L$ is said to be semicontinuous if every element $x \in L$ is normalable, i.e. if $\{x\}^{\perp}$ is a normal manifold for any $x \in L$. In [1] we proved the

THEOREM. A linear lattice is continuous if and only if it is semicontinuous and uniformly complete.

We used spectral theory to prove it. In this paper we will give another proof without use of spectral theory.

Let $L$ be a linear lattice. If $L$ is continuous, then $L$ is semicontinuous by Theorem 6.15 of [2] and uniformly complete by Theorem 3.3 of [2]. Thus we will prove the converse.

We suppose that $L$ is semicontinuous and uniformly complete. First we prove that if a sequence $0 \leqq x_{v} \uparrow_{v=1}^{\infty}$ is bounded, then there is $z \in L$ such that $\left[x_{v}\right] x \uparrow_{v=1}^{\infty}[z] x$ for $x \geqq 0$. If $x_{v} \leqq k$ for $v=1,2, \cdots$, then setting

$$
y_{n}=\sum_{v=1}^{n} \frac{1}{v}\left(\left[x_{v}\right]-\left[x_{v-1}\right]\right) k \text { for } n=1,2, \cdots,
$$

where $x_{0}=0$, we obtain a uniform Cauchy sequence $y_{n}(n=1,2, \cdots)$. Since $L$ is uniformly complete by assumption, there exists $z \in L$ such that $y_{n} \uparrow_{n=1}^{\infty} z$. Then $\left[y_{n}\right] x \uparrow_{n=1}^{\infty}[z] x$ for $x \geqq 0$ by Theorem 5.26 of [2] because

Received by the editors October 14, 1971.

AMS 1970 subject classifications. Primary 46A40.

Key words and phrases. Functional analysis, vector lattices.

(c) American Mathematical Society 1972 
$\{z\}^{\perp}=\left\{y_{n}: n=1,2, \cdots\right\}^{\perp}$. On the other hand, by Theorem 6.3 of [2] we have

$$
\left[y_{n}\right]=\sum_{v=1}^{n}\left(\left[x_{v}\right]-\left[x_{v-1}\right]\right)[k]=\left[x_{n}\right] \text { for } n=1,2, \cdots .
$$

Now we suppose that $0 \leqq x_{v} \downarrow_{v=1}^{\infty}$. For $\alpha \geqq 0$, since $\left(\alpha x_{1}-x_{v}\right)^{+} \uparrow_{v=1}^{\infty}$ and $\left(\alpha x_{1}-x_{v}\right)^{+} \leqq \alpha x_{1}$ for $\nu=1,2, \cdots$, there exists $z_{\alpha} \in L$ such that

$$
\left[\left(\alpha x_{1}-x_{v}\right)^{+}\right] x \uparrow_{v=1}^{\infty}\left[z_{\alpha}\right] x \text { for } x \geqq 0,
$$

as proved above. It is clear that $\left[z_{0}\right]=0$ and $\left[z_{\alpha}\right]=\left[x_{1}\right]$ for $\alpha>1$ because $\alpha>1$ implies $(\alpha-1) x_{1} \leqq\left(\alpha x_{1}-x_{v}\right)^{+} \leqq \alpha x_{1}$. Since

$$
\left(\alpha x_{1}-x_{v}\right)^{+} \leqq\left(\beta x_{1}-x_{v}\right)^{+} \text {for } \alpha \leqq \beta,
$$

we have $\left[z_{\alpha}\right] \leqq\left[z_{\beta}\right]$ for $0 \leqq \alpha \leqq \beta$. Since $\left[\left(\alpha x_{1}-x_{v}\right)^{+}\right] \leqq\left[z_{\alpha}\right]$, we have

$$
\left(\left[z_{\beta}\right]-\left[z_{\alpha}\right]\right)\left[\left(\alpha x_{1}-x_{v}\right)^{+}\right]=0 \text { for } \alpha \leqq \beta .
$$

Thus $\left(\left[z_{\beta}\right]-\left[z_{\alpha}\right]\right)\left(\alpha x_{1}-x_{v}\right)=-\left(\left[z_{\beta}\right]-\left[z_{\alpha}\right]\right)\left(\alpha x_{1}-x_{v}\right)^{-} \leqq 0$, i.e.

$$
\alpha\left[\left(z_{\beta}\right]-\left[z_{\alpha}\right]\right) x_{1} \leqq\left(\left[z_{\beta}\right]-\left[z_{\alpha}\right]\right) x_{v} \text { for } \alpha \leqq \beta \text { and } v=1,2, \cdots \text {. }
$$

We consider a double sequence $0=\alpha_{\mu, 0}<\alpha_{\mu, 1}<\cdots<\alpha_{\mu, n_{\mu}}(\mu=1$, $2, \cdots)$ such that $\alpha_{\mu, v}-\alpha_{\mu, v-1}<1 / \mu$ for $v=1,2, \cdots, n_{\mu}, \alpha_{\mu, n_{\mu}}>1$, and $\alpha_{\mu, v}\left(\nu=1,2, \cdots, n_{\mu}\right)$ is a partial sequence of $\alpha_{\mu+1, v}\left(v=1,2, \cdots, n_{\mu+1}\right)$. Setting

$$
y_{\mu}=\sum_{v=1}^{n_{\mu}} \alpha_{\mu, v-1}\left(\left[z_{\alpha_{\mu, \nu}}\right]-\left[z_{\alpha_{\mu, v-1}}\right]\right) x_{1} \quad \text { for } \mu=1,2, \cdots,
$$

we obtain a uniform Cauchy sequence $y_{\mu}(\mu=1,2, \cdots)$ because

$$
\left|y_{\mu}-y_{\rho}\right| \leqq(1 / \mu)\left[z_{\alpha_{\rho, n_{\rho}}}\right] x_{1}=(1 / \mu)\left[x_{1}\right] x_{1}=(1 / \mu) x_{1} \text { for } \rho \geqq \mu .
$$

Since $L$ is uniformly complete by assumption, there exists $y \in L$ such that $\lim _{\mu \rightarrow \infty} y_{\mu}=y$. Since

we have

$$
\alpha_{\mu, v-1}\left(\left[z_{\alpha_{\mu, v}}\right]-\left[z_{\alpha_{\mu, v-1}}\right]\right) x_{1} \leqq\left(\left[z_{\alpha_{\mu, v}}\right]-\left[z_{\alpha_{\mu, v-1}}\right]\right) x_{\rho},
$$

$$
y_{\mu} \leqq \sum_{v=1}^{n \mu}\left(\left[z_{\alpha_{\mu, v}}\right]-\left[z_{\alpha_{\mu, v-1}}\right]\right) x_{\rho}=\left[z_{\alpha_{\mu, n_{\mu}}}\right] x_{\rho}=\left[x_{1}\right] x_{\rho}=x_{\rho}
$$

because $\left[x_{\rho}\right] \leqq\left[x_{1}\right]$. Thus $y \leqq x_{\rho}$ for $\rho=1,2, \cdots$.

We suppose $0 \leqq z \leqq x_{v}$ for $v=1,2, \cdots$. Since $\left[\left(\alpha x_{1}-x_{v}\right)^{+}\right]\left(\alpha x_{1}-x_{v}\right)=$ $\left(\alpha x_{1}-x_{v}\right)^{+} \geqq 0$, we have

$$
\alpha\left[z_{\alpha}\right] x_{1} \geqq \alpha\left[\left(\alpha x_{1}-x_{v}\right)^{+}\right] x_{1} \geqq\left[\left(\alpha x_{1}-x_{v}\right)^{+}\right] x_{v} \geqq\left[\left(\alpha x_{1}-x_{v}\right)^{+}\right] z
$$


for $\nu=1,2, \cdots$, and hence $\alpha\left[z_{\alpha}\right] x_{1} \geqq\left[z_{\alpha}\right] z$. Thus

$$
\alpha\left(\left[z_{\alpha}\right]-\left[z_{\beta}\right]\right) x_{1} \geqq\left(\left[z_{\alpha}\right]-\left[z_{\beta}\right]\right) z \text { for } \alpha \geqq \beta,
$$

because $\left(\left[z_{\alpha}\right]-\left[z_{\beta}\right]\right)\left[z_{\alpha}\right]=\left[z_{\alpha}\right]-\left[z_{\beta}\right]$ by Theorem 5.24 of [2]. Since $\alpha_{\mu, v-1}+$ $1 / \mu \geqq \alpha_{\mu, v}\left(\nu=1,2, \cdots, n_{\mu}\right)$, we have

$$
\begin{aligned}
y_{\mu}+\frac{1}{\mu} x_{1} & \geqq \sum_{v=1}^{n_{\mu}} \alpha_{\mu, v}\left(\left[z_{\alpha_{\mu, v}}\right]-\left[z_{\alpha_{\mu, v-1}}\right]\right) x_{1} \\
& \geqq \sum_{v=1}^{n_{\mu}}\left(\left[z_{\alpha_{\mu, v}}\right]-\left[z_{\alpha_{\mu, v-1}}\right]\right) z=\left[x_{1}\right] z=z
\end{aligned}
$$

for $\mu=1,2, \cdots$ because $0 \leqq z \leqq x_{1}$ implies $[z] \leqq\left[x_{1}\right]$. Since $L$ is Archimedean, $\lim _{\mu \rightarrow \infty}(1 / \mu) x_{1}=0$, and we obtain $y \geqq z$. Therefore $L$ is continuous by definition.

\section{REFERENCES}

1. B. Anderson and H. Nakano, Semi-continuous linear lattices, Studia Math. 37 (1971), 191-195.

2. H. Nakano, Linear lattices, Wayne State Univ. Press, Detroit, Mich., 1966. MR 33 \#3084.

Department of Mathematics, Wayne State University, Detroit, Michigan 48202 